\title{
Performance Evaluation of a File Sharing DTN Protocol with Realistic Mobility
}

\author{
Armir Bujari, Claudio E. Palazzi, Daniele Bonaldo \\ Università degli Studi di Padova \\ Padova, Italy \\ \{abujari, cpalazzi\}@math.unipd.it
}

\begin{abstract}
Mobile Ad-hoc Networks (MANETs) exhibit unpredictable topology with heterogeneous node contact rates, high mobility and limited information. When dealing with such challenging environments, the mobility model has a key role in testing the performance and proving the expected behavior of routing algorithms. While synthetic mobility models have been largely used to measure quantitative aspects of routing protocols, they are not sufficient as they do not capture reliably the properties of movement in the real life scenarios. In this paper we present some experimentation done in THE_ONE simulation environment, involving our M2MShare, a peer-to-peer (P2P) delay tolerant file sharing platform for mobile networks. For testing purposes we have chosen the Working Day Movement (WDM) model which is shown to make a good approximation of human mobility patterns and provides the flexibility of configuring real life test scenarios.
\end{abstract}

Keywords-P2P; opportunistic file sharing; multimedia; DTN; MANET

\section{INTRODUCTION}

The evolution of device technology has led to reductions in cost, size and power consumption, which have enabled mobile devices to increase their complexity, processing capability and usability. Nowadays, mobile phones have evolved from simple voice communication means into powerful devices able to handle complex multimedia documents. It seems hence interesting to export a popular application such as Peer-to-Peer (P2P) file sharing into the new scenario of mobile networks. In this context, different from the classical wired P2P file sharing approach, mobile users could exchange multimedia data when in proximity of each other by establishing opportunistic, proximity-based P2P ad-hoc connections $[1,2,3]$.

However, when dealing with mobile networks, communication cannot always rely on a continuous end-to-end path between source and destination as in the wired Internet. As due to mobility, communication links are transient and short in time, in sparse mobile ad-hoc networks this path might not even exist. Moreover, considering proximity-based P2P file sharing as in our case, low node density could imply lower data population in the overlay network, which could undermine the utility of a P2P file sharing application.

Our M2MShare embodies a delay tolerant communication solution for ad-hoc P2P opportunistic networks comprised of smartphones [4]. It ports the Delay/Disruption Tolerant Networking (DTN [5]) paradigm into the mobile world, addressing the node density issue by providing means for an asynchronous data exchange similar to that of DTNs. The idea of a DTN is modelled in an infrastructure-less environment where both the source of the file request and the destination of the data are the same entities and where intermediary nodes (servants) can store-delegate-and-forward back the requested data toward the source. Our focus is on exploring a new mechanism that allows peers to expand their reach area to other local disconnected networks. M2MShare achieves this by leveraging on node mobility and periodic encounters among users even if they are not aware of this social proximity (e.g. commuters utilizing the same train every morning even if they do not know each other personally). In essence, content search/download is performed by both enquiring encountered nodes but also by delegating unsatisfied unaccomplished file search/download task to some of them. To avoid excessive transmission overhead, delegations are assigned only to nodes that are probably met again in the future (i.e., nodes that are frequently met).

This mobile opportunistic network could be comprised of human-operated mobile devices moving in restricted physical spaces, such as conferences, university campuses, refectories, clubs and in many other social settings. In essence, they are characterized by nodes with heterogeneous contact rates, high mobility and limited information. To this purpose, in order to provide quantitative and qualitative measurements of our software we have chosen a delay tolerant simulation environment called THE ONE [6]. To increase the trustworthiness of the experimental outcome we have included realistic day-by-day node movements through the Working Day Movement (WDM) model. This model is able to represent both the unpredictability of certain movements of users and the routine of other movements such as, for instance, the daily trip from home to work. This provides a good approximation of inter-contact times and contact durations, providing the flexibility for configuring real life test scenarios $[7,8]$.

In this work, we discuss M2MShare and provide a set of realistic simulation scenarios for opportunistic communications specifically designed for DTNs composed by smartphones. Our simulation scenarios include i) real life scenarios comprised of activities resembling human behavior; ii) quantitative and qualitative experimentation results showing that our M2MShare serves its purpose by outperforming other classical strategies in different metrics. 
The remainder of this paper is organized as follows. Section II summarizes background information related to our work. In Section III we discuss M2MShare by introducing operational details and protocol stack, describing the duties and responsibilities of each individual layer. Section IV provides some insights on the servant election strategy; also, we show different experimentation proving that the delegation technique serves its purpose. Finally, concluding remarks are given in Section V.

\section{BACKGROUND}

In this section we present some background information regarding the applicative domains concerning our work. We begin by stating some of the challenges encountered when deploying P2P on Mobile Ad-hoc Networks (MANETs), further proceeding in our discussion with the DTN communication model.

\section{A. P2P on MANETS}

Mobile devices are providing functionalities previously restricted to desktop computers. Such devices can interconnect using broadband wireless network interfaces. Local storage and computing capacity are sufficient for storing and playing highquality audio, movie files and complex multimedia presentations. Due to their low cost, they are growing in popularity and may at some time become the dominant mode by which users reach the Internet, in a way we could consider them as an opportunistic extension of the today's Internet. Consequently, they will have an important role in future P2P overlays. However, mobile networks have some important characteristics that differentiate them from the Internet and that affect their interaction with the classical P2P wired overlays.

The static connection approach employed by such systems in the wired case is not suitable for MANETs; this due to mobility disconnections are often to occur and maintenance overhead is not sustainable [9, 10]. Also, in sparse mobile environments, there can be no path between the source and the destination and moreover when considering our applicative scenario, low node density could mean lower data population in the overlay which could undermine the utility of a P2P file sharing application [2, 9]. For these reasons synchronous communications are not sufficient in such an environment and other communication approaches are needed.

Furthermore, mobile devices have energy issues, thus the need to preserve energy is essential $[10,11,25]$. Power management involves a combination of techniques, including network adapters that can trigger power resume of the host while offloading certain network activity and network protocols that reduce power consumption. In current overlay designs, a mobile peer that goes into power-saving mode is treated as a node that has left the overlay. Another, not less important characteristic that differentiate the wireless from the wired world is the communication technology. The wireless medium, as opposed to the wired communication technology, is known to be error-prone and bandwidth limited. Limited bandwidth alone could mean lower data quantity transferred, adding to this the mobility factor and low peer density we might need other ways of reliable synchronous or asynchronous data transfer.

Concluding, mobile environments face multiple challenges and traditional solutions for P2P over fixed networks may need to be redesigned when applied in the wireless mobile world.

\section{B. Delay/Disruption Tolerant Network (DTN)}

The TCP/IP protocol suite has been a great success at interconnecting communication devices across the Internet [13]. Yet, it is based on the assumptions of having connectivity primarily on wired links, continuously connected end-to-end devices, and low-delay paths between the sources and destinations.

Nowadays, with the growing number of devices equipped with wireless interfaces, users increasingly find themselves in different types of networking environments than the aforementioned ones. These environments, spanning from globally connected networks such as cellular networks or the Internet to the entirely disconnected networks of stand-alone mobile devices, impose different forms of connectivity. In particular, differently from the wired Internet communication, MANETs composed by smartphones are characterized by:

- Intermittent connectivity: connection links are transient and short in time due to mobility. TCP/IP is not suitable under such circumstances, other protocols are required.

- Long or variable delays: Propagation delays between nodes and variable queuing delays at nodes contribute to end-to-end delays that can defeat Internet protocols and applications that rely on quick return of acknowledgements or data.

- Asymmetric data rates: The internet supports moderate asymmetries of bidirectional data rate for users with cable TV or asymmetric DSL access. But if asymmetries are large, they defeat actual protocols.

- High error rates: Bit errors on links require correction (added redundancy and processing time) or retransmission of the entire packet. For a given linkerror rate, fewer retransmissions are needed for hopby-hop than for end-to-end retransmission.

A DTN originally thought and conceived for communication in outer space, is an overlay of networks, including the Internet. DTNs support interoperability of networks by accommodating long delays between and within them, and by interfacing different network types. In providing these functions, DTNs accommodate the mobility and limited power of evolving wireless communication devices (smartphones). They employ a store-and-forward message switching (Fig. 1); whole messages or pieces of such messages are forwarded from a storage place on one node to a storage place on another node, along a path that eventually reaches destination. 

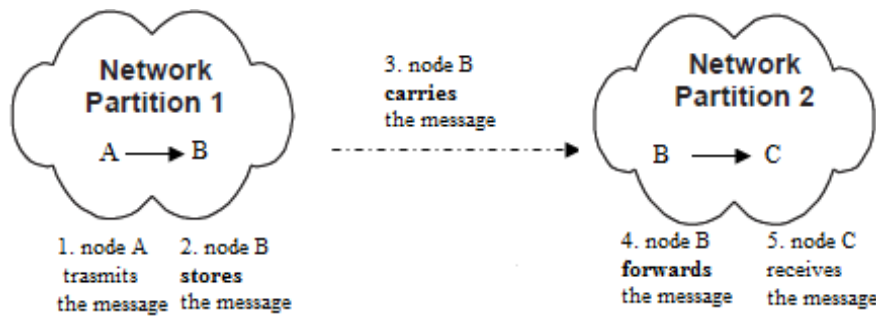

Figure 1. Store-and-forward packet switching between network nodes.

Another peculiarity of DTNs is that their routers need persistent storage for their queues as opposed to Internet routers that use short-term storage provided by memory chips. This, for the following reasons:

- a communication link to the next hop may not be available for a long time;

- $\quad$ one node in a communicating pair may send or receive data much faster or more reliably than the other node;

- a message, once transmitted, may need to be retransmitted if an error occurs at an upstream (toward destination) node or link, or if an upstream node declines acceptance of a forwarded message.

The DTN paradigm is still an active area of research and a lot of other features would be worth mentioning but are outside of the scope of this work (for more insights refer to [14]). However, as we discuss in this paper, it represents an interesting option for opportunistic communication among smartphones.

\section{M2MSHARE}

The evolution of device technologies and the end-user trends to shift toward wireless technology have made possible the exchange and playback of complex multimedia content between mobile devices. As P2P networks were initially designed and conceived for the wired Internet such networks do not perform well on mobile challenged environments, this due to fundamental differences between the two. Mobility and low node density require a revision of networking protocols toward a delay tolerant approach. In this section we expose some operational details of M2MShare, the application we designed and implemented to demonstrate the opportunistic DTN approach in mobile to mobile multimedia content sharing.

\section{A. Modus operandi}

M2Mshare uses Bluetooth to create a P2P overlay networks, which would allow the automatic exchange of multimedia content among smartphones [15]. Our software automatically initiates a search by broadcasting the user's query request toward other Bluetooth enabled devices operating the program. Once the answer/s is received, data found to match the criteria will be automatically requested for download. All this process is done without the user mediation; the user only needs to specify the search terms, configurable through the user interface and chose from the matched content list. Key features of M2MShare are its ease of use and autonomy, once the initial preferences are set up no further user interaction is needed and the user can find downloaded files directly on her/his phone, when done.

Given that energy consumption is a problem for handheld devices the notion of active sessions was introduced. An active session is a period of time in which the software is functional and performs its duties and such periods are configurable through the graphical user interface. This way, the user can set the application to look for requested contents only in certain periods of the day; for instance, when commuting or during lunch time in the cafeteria, so as to be active only when it could be useful since the high number of other peers around.

As stated earlier, we leverage on mode mobility to reach data content on other local disconnected networks. This is achieved by introducing an asynchronous communication model, store-delegate-and-forward where a peer delegates and unaccomplished/unsatisfied task to other peers in the overlay network. Delegating tasks to all encountered peers is energy and bandwidth consuming. Also, it would be useless to assign tasks to peers that will never be met again in the future. To avoid this M2MShare exploits social relations and delegates tasks only to frequently encountered peers, peers whom are expected to be encountered again in the future (refer to Section IV-A).

We could say that we exploit social relations among users to determine possible task delegates. This is not a new approach as context and social relations are already studied in opportunistic data transmission [16]. Yet, we utilize even unknown social relations by connecting users that have to pass by the same geographical location at the same time frequently enough. To assume that a candidate for task delegation could be met again in the future (so that she/he will be able to deliver contents possibly found), we use as an heuristic the history of previous encounters.

\section{B. Software Architecture}

We now introduce the protocol stack implemented by M2MShare from a top-down perspective and give a brief description for each individual layer.

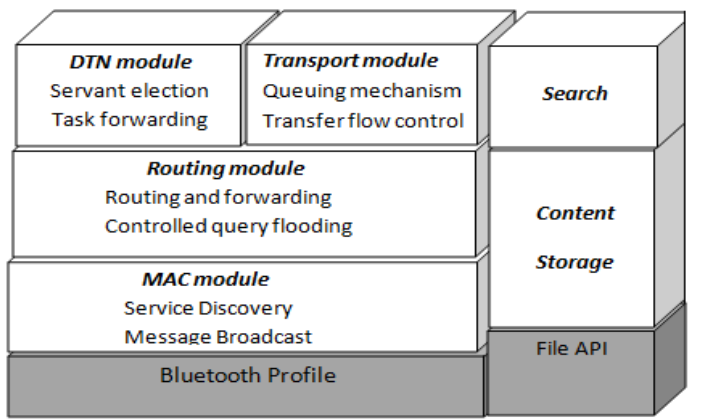

Figure 2. M2MShare peer architecture. Blocks shown in gray are proprietary.

Search module. This module comprises the indexing strategy for utilizing various query models: from simple keywords to range queries.

DTN module. This module is responsible for servant election and hence for task delegation. Studies in routing algorithms for challenging environments such as MANETs 
have a social dimension built in $[17,18]$; knowing that behavior patterns exist allows better routing decisions to be made. We exploited the fact that certain users frequently becomes in proximity of each other (e.g. by taking the same bus in the morning, by eating in the same cafeteria at lunch, etc.) in order to dynamically build a DTN path from source to destination.

Transport module. It provides the task queuing mechanism and task lifecycle management. These are important features when utilizing mobile devices that have resource constraints. An important part of this module is the communication protocol for data packet exchange among nodes. Also, it provides a smart file division strategy, which allows for parallel and hence faster download while avoiding redundancy on downloaded data.

Routing module. This module provides message forwarding capabilities to our overlay network and implements a controlled flooding technique alike AODV [12]. It implements the overlay organization, i.e. connection establishment and maintenance, as done by Optimized Routing Independent Overlay (ORION [9]) protocol.

MAC module. It provides service discovery and message broadcast facility for peers in our network. The heart of this module is a fundamental service called PresenceCollector which periodically gathers presence information [4] about inreach area devices so as to determine which nodes are frequently met and have hence a reasonable expectation to be met again in future (these nodes will be used as servant to propagate file queries or downloads).

\section{SERVANT ELECTION}

M2MShare implements an asynchronous communication model between peers, where a client peer can delegate an unsatisfied, unaccomplished task to a servant peer. While in DTNs there are pre-deployed entities that store-and-forward data along the destination path (routers), M2MShare achieves this functionality in an infrastructure-less environment, where this forward route is established dynamically along the path to destination. In this section we state the underlying assumptions behind the DTN module design and show some experiments which demonstrate that the delegation technique serves its purpose.

\section{A. Frequently encountered device}

While research into routing in mobile environments is not new, researchers have for many years assumed node encounters to be random. In reality, mobile nodes are of course used by people, whose behaviors are better described by social models. This opens up new possibilities for routing, since the knowledge of behavior patterns allows better routing decisions to be made $[17,18]$. In our work we exploit this idea of social relations between users operating mobile wireless devices and provide a proof of concept implementation, adopting a DTN type solution for the mobile disconnected networks.

Delegating an unaccomplished task to all the peers in the established overlay network is bandwidth and energy consuming therefore a criterion is needed to choose one peer instead of others in order to decrease the number of transmissions when possible. It is sound to delegate tasks to peer devices operated by users whom we expect to encounter again in the future. In fact it would be useless to assign a task to a node that will never be met in the future. As a heuristic to predict future probability of encountering a node we use the frequency of past encounters.

In the actual implementation, a servant device is a frequently encountered device and the concept of frequently encountered changes in time, adapting to the observed dynamics. This because the contact rate of a single device operating M2MShare might vary from day to day. Moreover, some devices frequently encounter many other devices, while others encounter a small number of them. In the first case we would want to higher the expectations of a frequently encountered device in order to choose the best devices from those repeatedly encountered. In the second case, in order to have a certain number of frequently encountered devices we should be less selective by lowering the system expectations (parameters). The servant election algorithm takes into consideration these cases and tunes the parameters accordingly to the observed history.

\section{B. Delegation efficiency}

In this section we compare the efficiency of our system (M2MShare) employing delegations against two other systems using different strategies:

- No_delegation: system which does not employ delegations and file exchange is initiated only when a peer holding the requested data file is found in reach area of file requester.

- Delegation_to_all: system employing delegations but instead employs the trivial technique where missing tasks are delegated to each encountered peer.

The metric we study is the found time $\left(F_{t}\right)$ for a generic data file which is the time interval between the first delegation made and the time an output return for that specific file is received. If no delegations are made and the first file request is satisfied by a direct file possessor the $F_{t}$ is equal to zero. For each of the above scenarios we also measure the:

- number of delegations used: representing the number of tasks the requestor peer has delegated for that particular data download;

- percentage of completed task: representing the number of delegated tasks completed (output returned) over all delegated tasks;

- total data transferred: referring to the quantity of data traffic exchanged between servant peers trying to satisfy a delegated task, file possessor peers and the data forwarding quantity toward the requestor node.

To this purpose we implemented the three protocols in THE_ONE. For sake of simplicity, delegations are one hop only, i.e., a servant peer cannot further delegate the task to other frequently encountered peers of its own. 


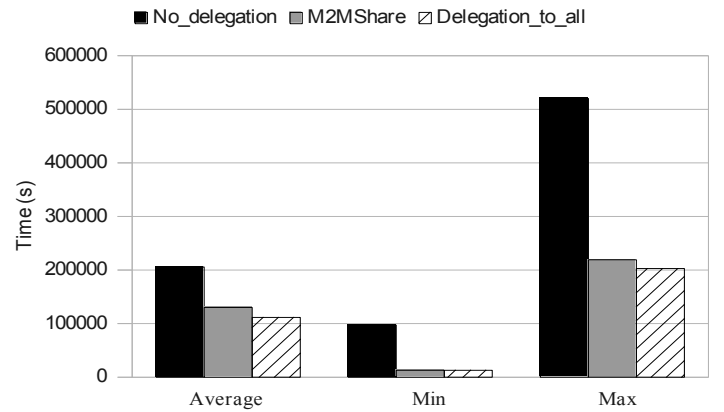

Figure 3. Average, min. max found time employed by each strategy in finding the required data file.

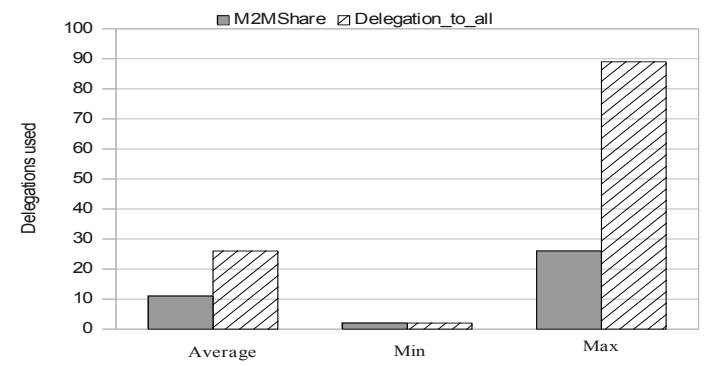

Figure 4 - Average, min, max number of delegations employed by each delegation strategy.

Also the software is always operational, there is only one active session configured that covers all day user activity. For the simulations, as anticipated, we have used the Working Day Movement (WDM) model with the Helsinki map available in THE ONE. Each scenario has a population of 1000 nodes which emulate people operating M2MShare and are involved in their daily activities according to the cycle, home-workevent-home. A node at home is inactive, thus the software is not operative. Nodes are uniformly distributed between the available districts of the map and the simulation time is set to seven working days, during which there are seven full cycles home-work-event-home. The data file the user is looking for is fixed with a size of 3.0 MB and it is, at the beginning of every simulation, in possess of 50 randomly chosen and distributed nodes of the population.

We repeat each of the simulation scenarios 40 times in order to achieve more accurate results, independent of the initial positioning of the requestor peer in search for that particular data file. Each scenario is run using a different random seeds to initialize the movement model and for every seed the simulation is repeated using the three compared protocols.

In Fig. 3 it is possible to see the advantage, in terms of found time, in using the delegation technique instead of not using it. The two systems employing delegations find the required file in less time in each simulation run at the expense of higher overhead in terms of bandwidth due to delegations. The system employing delegations to all encountered peers gets a better result on average, but at a cost of a higher number

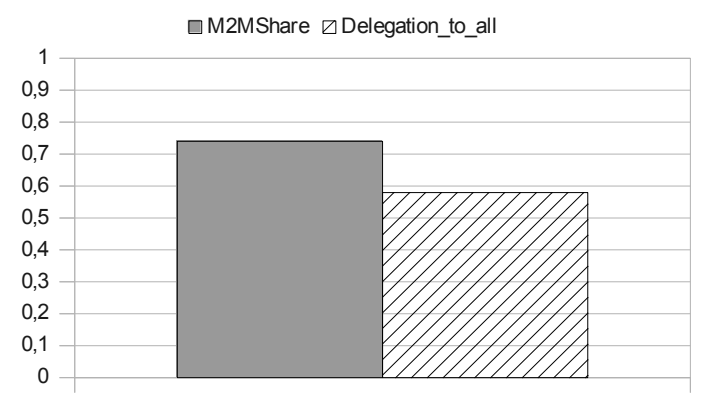

Figure 5. Percentage of completed previously delegated tasks against the number of overall delegations employed.

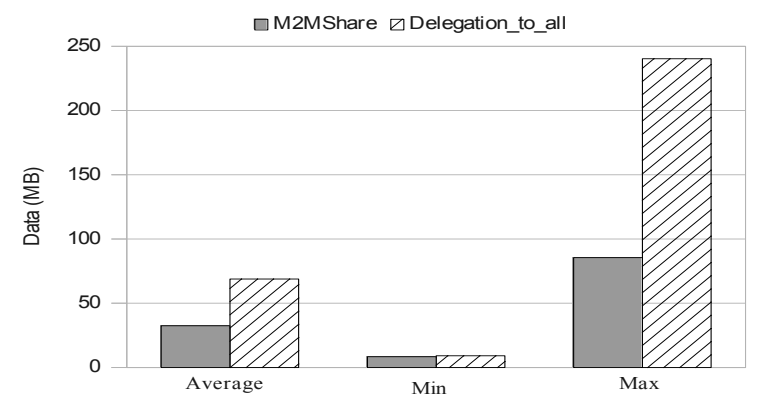

Figure 6. Average, min, max transferred data amount employed in each delegations technique.

of delegated tasks (Fig. 4). A higher number of delegated tasks imply more bandwidth used for searching the data file and potentially retrieving (if found) and forwarding it toward the requestor.

Fig. 4 makes a comparison between the two systems employing delegations by showing the number of overall employed task delegations till file download or simulation time expires. It is easy to see that M2MShare uses fewer delegations while achieving a higher percentage of completed delegated tasks (Fig. 5). This outcome is due to a conservative delegation strategy employed by M2MShare in delegating unsatisfied, unaccomplished tasks only to frequently encountered peers (servants). Since we do not have any means to evaluate the ability of one servant to satisfy a file request what we do is delegate to encountered peers whom is expected to be encountered again in the future. The Delegation to all strategy contributes to higher overhead also due to completed tasks, ready to be returned toward the requestor that unfortunately expire and are discarded before having the chance of encountering the data file requestor.

In Fig. 6 is shown the comparison between M2MShare and Delegation_to_all strategies in terms of transferred data quantities till file download or simulation time ends. It is straightforward to notice the higher overhead in terms of data transmissions introduced by delegating to all encountered peers whether M2MShare reduces the exchanged data quantity while still achieving the goal of acquiring the requested file. From the above results it is obvious that the delegation strategy serves its purpose by extending a peer reach area to other mobile disconnected networks where data content might be available therefore reducing the found time of a desired content. 
Although this strategy introduces an overhead in terms of bandwidth usage, computation and power consumption we control these side effects by delegating only to frequently encountered peers whom are expected to be encountered again in the future.

\section{CONCLUSION}

End-user trends to shift toward wireless technologies and the device technology evolution has opened new application scenarios for the mobile device world. These challenging environments have fundamental characteristics that differ from the wired Internet, from mobility to low node density and energy preservation issues which need to be addressed.

The key ingredient in demonstrating the utility and good design choices of a routing algorithm is the adopted mobility model. In this paper we presented some experimentation results devised for M2MShare. To this purpose, we adopted THE_ONE, a delay tolerant networking simulation environment along with the Working Day Movement (WDM) model which makes a good approximation of human mobility patters in terms of inter-contact and contact duration also providing the flexibility of configuring real life testing scenarios.

We showed by experimental means that the delegation technique serves its purpose by reducing the found time of a generic file while reducing the number of overall transmissions. Possible extensions of this work include the design of a file division strategy and local congestion control to increase the quantity of file downloaded per time unit $[19,20]$. Moreover, it would be interesting to test possible support of our solution to entertainment applications such as gaming, alert message propagation, and vehicular applications [21, 22, 23, 24]. Finally, to this purpose we are currently planning a comprehensive set of simulations through NS2 which makes possible the testing of the entire protocol layers devised for our software.

\section{REFERENCES}

[1] C. E. Palazzi, "Buddy-Finder: A Proposal for a Novel Entertainment Application for GSM", in Proc of IEEE International Workshop on Networking Issues in Multimedia Entertainment 2004 (IEEE NIME 2004), GLOBECOM 2004 Dallas, TX, USA, Nov 2004

[2] A. Di Ferdinando, P. McKee, A. Amoroso, "A Policy Based Approach for Automated Topology Management of Peer To Peer Networks and a Prototype Implementation", in Proc of the 4th IEEE International Workshop on Policies for Distributed Systems and Networks, Como, Italy, Jun 2003.

[3] S. Jung, U. Lee, A. Chang, D.-K. Cho, M. Gerla, "BlueTorrent: Cooperative Content Sharing for Bluetooth Users", Pervasive and Mobile Computing 3(6): 609-634, 2007.

[4] C. E. Palazzi, A. Bujari, "A Delay/Disruption Tolerant Solution for Mobile-to-Mobile File Sharing", in Proc of the $3^{\text {rd }}$ IFIP/IEEE Wireless Days 2010, Venice, Italy, Oct 2010.

[5] V. Cerf, S. Burleigh, A. Hooke, L. Torgerson, R. Durst, K. Scott, K. Fall, H. Weiss, Delay-Tolerant Networking Architecture, http://tools.ietf.org/html/rfc4838 Apr 2007.

[6] A. Keränen, J. Ott, T. Kärkkäinen, "The ONE simulator for DTN protocol evaluation", in: Proceedings of the $2^{\text {nd }}$ International Conference on Simulation Tools and Techniques, Rome, Italy, 2009.
[7] F. Ekman, A. Keränen, J. Karvo, J. Ott, "Working Day Movement Model", in: Proceeding of the 1st ACM SIGMOBILE workshop on Mobility models, NY, USA, 2008.

[8] M. Musolesi, C. Mascolo, "A community based mobility model for ad hoc network research", In Proc of the $2^{\text {nd }}$ international workshop on Multi-hop ad hoc networks: from theory to reality, Florence, Italy, May 2006.

[9] A. Klemm, C. Lindemann, O. Waldhorst, "A Special-Purpose Peer-toPeer File Sharing System for Mobile Ad Hoc Networks", in Proc of IEEE Semiannual Vehicular Technology Conference 2003 (IEEE VTC 2003, Orlando, FL, USA, Oct 2003.

[10] J. Wu, "Theoretical and Algorithmic Aspects of Sensor, Ad Hoc Wireless, and Peer-to-Peer Networks", 2005, 845 - 865, 2005.

[11] S. Gurun, P. Nagpurkar, and B. Y. Zhao, "Energy consumption and conservation in mobile peer-to-peer systems", in: Proceedings of the 1st international workshop on Decentralized resource sharing in mobile computing and networking, Los Angeles, Jul. 25-25, 2006.

[12] C. E. Perkins, E. M. Belding-Royer, I. D. Chakeres, "Ad hoc OnDemand Distance Vector (AODV) Routing", IETF Internet Draft, available at draft-perkins-manet-aodvbis-01.txt. January 2004.

[13] W. R. Stevens, TCP/IP Illustrated, Volume 1- The Protocols, AddisonWesley, April 2000.

[14] Delay Tolerant Networking Research Group, available at http://www.dtnrg.org/wiki.

[15] Bluetooth 1.1: Connect Without Cables, Second Edition, Prentice Hall, 2001

[16] C. Boldrini, M. Conti, F. Delmastro, A. Passarella, "Context- and SocialAware Middleware for Opportunistic Networks". Journal of Network and Computer Applications, 33(5):525-541, 2010.

[17] I. Rhee, M. Shin, S. Hong, K. Lee, S. Chong, "Human Mobility Patterns and Their Impact on Delay Tolerant Networks", ACM HotNets VI, Atlanta, GA, USA, November 2007.

[18] S. Samal, Mobility Pattern Aware Routing in Mobile Ad Hoc Networks 2003, Master's Thesis, available at http://scholar.lib.vt.edu/these.

[19] G. Marfia, P. Lutterotti, S. Eidenbenz, G. Pau, M. Gerla, "Fair MultiMedia Streaming in Ad Hoc Networks through Local Congestion Control"', Proc. 11-th ACM International Conference on Modeling, Analysis and Simulation of Wireless and Mobile Systems (MSWIM'08), Vancouver, Canada, Oct 2008.

[20] G. Marfia, G. Pau, E. Giordano, E. De Sena, M. Gerla, "VANET: On Mobility Scenarios and Urban Infrastructure. A Case Study", in Proc. 1st IEEE INFOCOM Workshop on Mobile Networking for Vehicular Environments (MOVE'07), Anchorage, Alaska, USA, May 2007.

[21] S. Ferretti, M. Roccetti, "Fast Delivery of Game Events with an Optimistic Synchronization Mechanism in Massive Multiplayer Online Games", in Proc. 2005 ACM International Conference on Advances in Computer Entertainment Technology, Valencia, Spain, Jun 2005.

[22] C. E. Palazzi, M. Roccetti, S. Ferretti, G. Pau, M. Gerla, "Online Games on Wheels: Fast Game Event Delivery in Vehicular Ad-hoc Networks", in Proc. of 3rd IEEE International Workshop on Vehicle-to-Vehicle Communications 2007 (V2VCOM 2007) - IEEE Intelligent Vehicles Symposium 2007, IEEE Intelligent Transportation Systems Society, Istanbul, Turkey, Jun 2007.

[23] M. Roccetti, M. Gerla, C. E. Palazzi, S. Ferretti, G. Pau, "First Responders' Crystal Ball: How to Scry the Emergency from a Remote Vehicle", in Proc. of the 1st IEEE International Workshop on Research Challenges in Next Generation Networks for First Responders and Critical Infrastructures (NetCri 07) - 26th IEEE International Performance Computing and Communications Conference (IPCCC 2007), New Orleans, LA, USA, IEEE Computer Society, Apr 2007.

[24] A. Sardouk, S. M. Senouci, N. Achir, K. Boussetta, "Assessment of MANET Broadcast Schemes in the Application Context of Multiplayer Video Games", in Proc. Of the 6th Annual Workshop on Network and Systems Support for Games, Netgames, Melbourne, Australia, Sep 2007.

[25] A. Kaiser, N. Achir, K. Boussetta, "Multiplayer Games over Wireless Ad hoc Networks: Energy and Delay Analysis", in Proc. of Ubiquitous Multimedia Services and Applications workshop (UMSA), StPetersburg, Russia, Oct 2009. 